Voix et Images

volxetimages

\title{
Entretien avec Jacques Poulin
}

\section{Jean-Pierre Lapointe et Yves Thomas}

Volume 15, numéro 1 (43), automne 1989

Jacques Poulin

URI : https://id.erudit.org/iderudit/200811ar

DOI : https://doi.org/10.7202/200811ar

Aller au sommaire du numéro

Éditeur(s)

Université du Québec à Montréal

ISSN

0318-9201 (imprimé)

1705-933X (numérique)

Découvrir la revue

Citer ce document

Lapointe, J.-P. \& Thomas, Y. (1989). Entretien avec Jacques Poulin. Voix et Images, 15(1), 8-14. https://doi.org/10.7202/200811ar d'utilisation que vous pouvez consulter en ligne.

https://apropos.erudit.org/fr/usagers/politique-dutilisation/ 


\section{Entretien avec Jacques Poulin}

\section{par Jean-Pierre Lapointe et Yves Thomas, université Trent}

V. \& I. - Jacques Poulin, quand on a lu vos romans, on a l'impression de bien vous connaître sans vous avoir jamais rencontré, tellement vous semblez être vous-même le personnage central. Y a-t-il de l'ironie derrière cette apparente ouverture sur vous-même?

J. Poulin - Il n'y a pas beaucoup d'ironie, je crois. Quand j'ai commencé à écrire, j'ai donné instinctivement au personnage principal mon âge, mes goûts et un travail semblable au mien, et j'ai fait la même chose dans les livres qui ont suivi. D'un livre à l'autre, le personnage a vieilli en même temps que moi. Dans le livre que je viens de terminer (et qui n'a pas encore paru), il est un peu plus jeune que moi, mais c'est parce que j'ai horreur de vieillir...

V. \& I. - Dans le Cœur de la baleine bleue, Noël se demande si, en écrivant des histoires, on peut être tout aussi honnête que dans une autobiographie.

J. Poulin - Je trouve que Noël a tort de se poser cette question. Ce qui compte, dans la fiction, ce n'est pas l'honnêteté, mais plutôt la vraisemblance. On part des choses que l'on a vécues et on les transforme, mais ce n'est pas tellement pour se cacher: c'est pour que l'histoire ait l'air d'être vraie.

V. \& I. - Vous dites qu'il faut partir du vécu... Est-ce qu'on ne peut pas faire de la fiction sans passer par les référents autobiographiques?

J. Poulin - Bien sûr. Certains auteurs le font - Hubert Aquin par exemple mais c'est plutôt rare et certainement plus difficile. Au fond, le plus important, c'est d'écrire sur les choses qu'on connaît le mieux. C'est en tout cas la meilleure façon d'éviter les erreurs.

V. \& I. - Dans plusieurs de vos romans, il y a une quête des origines, un mouvement de retour vers l'enfance afin de la démythifier. Mais les données autobiographiques qui se rapportent à l'enfance sont très peu nombreuses: vous ne parlez pas de votre père, de votre mère, de votre famille, de votre Beauce natale.

J. Poulin - Parler directement de ma famille et de mon enfance me semblerait une chose tout à fait indécente. L'enfance est pour moi une sorte de territoire sacré que je n'ai pas envie d'ouvrir à tout le monde. Il m'arrive d'en parler, mais c'est d'une manière allusive, et encore... À chaque fois, j'ai l'impression d'être au bord de l'indécence. Pourtant, quand c'est un autre écrivain qui parle de son enfance, ça ne me choque pas du tout.

V. \& I. - Dans votre œuvre, il y a tout de même la quête de l'enfance.

J. Poulin - Oui. 
V. \& I. - Pourquoi? C'est une conjuration? Une sorte d'exorcisme?

J.Poulin - Je n'en sais rien. Je suis très peu capable de réfléchir sur ce que j'ai écrit, et je n'aime pas beaucoup mes livres: ils sont trop éloignés de l'idéal que je veux atteindre.

V. \& I. - À quel moment s'est dessinée votre carrière d'écrivain? Est-ce que vous écriviez jeune?

J. Poulin - Aussi loin que je me rappelle, j'ai toujours eu l'envie d'écrire - une envie très vague au début, qui s'est précisée par la suite. Mais j'ai retardé le moment de m'y mettre, sans doute parce que j'avais peur. Ainsi, j'ai prolongé mes études: après avoir étudié la psychologie à l'université Laval, je suis allé en Lettres et je me suis inscrit à tous les cours portant sur le roman, dont un très bon cours du professeur Thorburn sur le roman anglais et américain. C'était inconsciemment une façon de me rapprocher de l'écriture romanesque. À la fin de mes études en Lettres, je me suis rendu compte que, à 27 ans, je n'étais plus tout jeune et qu'il me fallait commencer à écrire, alors je me suis donné deux ans pour écrire un premier roman. J'envoyais les chapitres un à un à Réginald Martel qui, à cette époque, n'était pas chroniqueur littéraire; il me lisait très patiemment, proposait des corrections et m'encourageait. Au bout de deux ans, le roman étant terminé, je l'ai fait parvenir aux Éditions du Jour où il a été lu par André Major et publié par Jacques Hébert.

V. \& I. - Dans vos romans, vous vous attardez longuement à décrire certains lieux: d'abord la ville de Québec, ensuite le circuit du mont Tremblant, l'île Madame et les villes américaines. Est-ce que les lieux ont beaucoup d'importance pour vous?

J. Poulin - Je place toujours mes personnages dans les endroits que je connais le mieux, comme le Vieux-Québec où j'ai vécu longtemps, ou encore le bord du fleuve où j'ai passé plusieurs étés. Dans Volkswagen Blues, où les personnages se déplacent de la Gaspésie à la Californie, il s'agit d'un parcours que j'ai fait moi-même à plusieurs reprises et j'ai séjourné à San Francisco pour écrire les derniers chapitres.

V. \& I. - Vous avez une prédilection pour Québec?

J. Poulin - C'est l'endroit où j'ai habité le plus longtemps.

V. \& I. - Comme vous êtes en Europe depuis trois ans, est-ce que l'action de votre demier roman se passe sur ce continent?

J. Poulin - Non. Ce roman, qui s'appelle le Vieux Chagrin, a été commencé il y a cinq ans, au moment où j'étais à Key West. Ensuite, j'en ai écrit un bout à Cap-Rouge, un autre bout dans le vieux camper Volkswagen avec lequel je visitais plusieurs pays d'Europe, et je l'ai terminé et corrigé en France. L'action se déroule au bord du fleuve, non loin de Québec, sauf deux ou trois chapitres qui se passent en Europe. En fait, les livres sont en retard sur ce qu'on vit, à cause du temps qu'on met à les écrire; dans mon cas, il faut cinq ou six ans.

V. \& I. - Vous méditez longuement sur certaines idées, certains mots? 
J. Poulin - J'écris d'une manière instinctive, intuitive, sans trop savoir où je vais. C'est une façon de travailler qui demande beaucoup de temps, car elle donne lieu à des tâtonnements, des essais, des erreurs, des blocages. Il serait plus simple et plus logique de faire un plan et de le suivre, mais...

V. \& I. - Et vous partez de quoi? D'une image? Noël, dans le Cœur de la baleine bleue, dit une image qu'il faut laisser pourrir en soi.

J. Poulin - Une image, une émotion, je ne sais pas. De toute manière, au début, il n'y a presque rien. Ordinairement, il y a un personnage. Un jour, ce personnage rencontre quelqu'un, alors je les observe, $j$ 'essaie de voir ce qu'ils vont faire: c'est le début d'une piste et je ne sais pas où ça s'en va. Il y aura de longs détours que je devrai peut-être enlever au moment des corrections.

V. \& I. - Est-ce que vous faites beaucoup de corrections?

J. Poulin - Oui, beaucoup. Au début, je mettais deux ans pour écrire un livre. Maintenant, je mets à peu près cinq ans. Je fais un premier brouillon; ensuite, j'en refais un deuxième. Le reste, c'est de la correction. J'enlève des choses.

V. \& I. - Alors vous raturez plus que vous n'ajoutez?

J. Poulin - Quand je corrige, je n'ajoute presque rien: j'enlève. J'enlève à peu près le tiers du texte, surtout des adverbes et des adjectifs, mais parfois des phrases, des paragraphes et même des chapitres entiers. Comme j'ai toujours peur d'être trop long et d'ennuyer le lecteur, j'enlève tout ce qui ne me paraît pas essentiel.

V. \& I. - C'est très flaubertien, tout ça.

J. Poulin - En tout cas, ce n'est pas très économique!

V. \& I. - Vous aimeriez mieux être comme «l'écrivain idéal» de Volkswagen Blues, celui qui écrit d'un jet, pendant trois jours et trois nuits de frénésie qui le vident, l'épuisent et le laissent dans un état comateux? C'est un peu la manière dont Kerouac écrivait.

J. Poulin - Ce serait sûrement plus agréable.

V. \& I. - Le genre d'écrivain qui revient le plus souvent à travers vos personnages, c'est l'artisan. Par exemple, Teddy Bear qui fignole, qui travaille d'une façon très méticuleuse. Mais vos récits rendent compte aussi de la technologie. Dans les Grandes Marées, précisément, ces deux mondes se rencontrent. Avez-vous voulu montrer ce contraste?

J. Poulin - Bien sûr, mais je n'ai vraiment aucun talent pour analyser mes histoires. Je préfere laisser ce travail à des gens plus compétents. Déjà, je n'aime pas beaucoup mes livres, alors si en plus il faut que je les analyse, je vais me mettre à les détester complètement.

V. \& I. - Est-ce que vous pourriez parler un peu du livre que vous venez de terminer?

J. Poulin - Avec plaisir. C'est un court roman, une histoire d'amour intitulée le Vieux Chagrin, comme je l'ai dit, dont l'action se passe dans une vieille 
maison en bois isolée au bord du fleuve. Les personnages sont un homme dans la quarantaine, une femme appelée Marika, une autre femme, une très jeune fille et un chat nommé Chagrin. J'avais l'impression, en écrivant cette histoire, qu'elle était une sorte de prolongement adulte de Jimmy.

V. \& I. - C'est intéressant qu'il y ait dans vos derniers romans une sorte de retour à la fable, à quelque chose qui n'est pas une histoire moderne, sans que le monde moderne en soit exclu.

J. Poulin - Pour éviter que ce livre soit trop fermé sur lui-même, et qu'il ressemble aux Grandes Marées, j'ai essayé d'ouvrir des fenêtres sur le monde extérieur, mais aussi sur l'intérieur. J'espère qu'on sentira passer plus d'émotions dans cette histoire que dans les précédentes.

V. \& I. - Pour Jimmy, vous avez reconnu l'influence de Salinger. Est-ce que cette influence est confortée dans ce Jimmy adulte?

J. Poulin - Non. Je crois que c'est un livre qui ne doit rien à personne.

V. \& I. - Cependant, il y a dans votre œuvre une thématique qui rappelle beaucoup la littérature américaine moderne, certains thèmes qui sont assez communs aux auteurs américains, comme l'errance, le culte du héros, le paradis perdu, la recherche du passé.

J. Poulin - Est-ce que ça ne vient pas tout simplement du fait que je suis né et que j'ai vécu en Amérique du Nord? Ou peut-être que ça vient de mes lectures?

V. \& I. - Vos lectures de jeunesse, c'étaient surtout des auteurs américains?

J. Poulin - Dans ma jeunesse, mes lectures étaient très variées; je lisais tout et n'importe quoi. Mais avec le temps, les auteurs américains ont pris de plus en plus de place: Hemingway, Salinger, Vonnegut, Brautigan et maintenant Raymond Carver. J'aime bien cette façon d'écrire très concrète, avec peu d'épithètes, des phrases courtes sans inversions, la simplicité absolue, le dépouillement, la sobriété. En général, ce sont les Américains qui font ça le mieux, tandis qu'en littérature française, on cherche plutôt une phrase élégante, savante, harmonieuse.

V. \& I. - Y a-t-il un roman d'Hemingway qui a été déterminant pour vous?

J. Poulin - J'ai une grande affection pour le Vieil Homme et la mer et Paris est une fête; j'aime tous ses livres, y compris le tout dernier, le Jardin d'Éden, un manuscrit resté inachevé. Mais ce que j'aime le plus chez cet auteur, à part sa façon d'écrire, c'est son honnêteté absolue à l'égard de son métier.

V. \& I. - Il travaillait de façon très disciplinée, tous les jours.

J. Poulin - Oui. Il avait beaucoup de courage et d'énergie. Pour moi, c'était avant tout un écrivain et non pas un chasseur de fauves.

V. \& I. - Maintenant, dans les commentaires sur vos livres, on parle immanquablement de tendresse... à tel point qu'on se demande s'il est pertinent d'en parler ici. Mais il y a quand même une quête du bonheur à travers tout ça, comme si la tendresse était un premier pas vers le bonheur. Est-ce que pour vos personnages le bonheur est accessible ou est-ce tout simplement une illusion? 
J. Poulin - La recherche du bonheur, c'est justement le thème du Vieux Chagrin, alors si vous voulez bien attendre la publication....

V. \& I. - Est-ce que ce dernier roman a exigé autant de recherches que Volkswagen Blues?

J. Poulin - Pas autant de recherches, mais presque autant de travail, même si le livre fait moins de deux cents pages. Comme pour Volkswagen Blues, j'ai dû faire deux brouillons et, au moment des corrections, j'ai coupé le tiers du texte. Mais surtout, j'ai travaillé plus que d'habitude sur l'écriture; j'ignore si ça se verra: c'est généralement une chose qui passe inaperçue.

V. \& I. - Le style est changé?

J. Poulin - J'ai essayé de garder un style très dépouillé, mais cette fois en allongeant les phrases et en variant leur forme.

V. \& I. - Une chose étonnante, c'est que vous n'écrivez pas tellement sur le Québec. Vos personnages vivent à une époque très marquée par la quête de l'identité collective, la question de l'indépendance, mais ils n'en sont pas préoccupés. Est-ce qu'on peut dire, néanmoins, qu'il y a une sorte de discours social et politique, un discours allégorique, au deuxième niveau, dans vos romans?

J. Poulin - Dans certaines de mes histoires, les Grandes Marées notamment, il y a des éléments de critique sociale, mais ça ne compte pas beaucoup à mes yeux. Comme romancier, je ne veux pas me placer d'un point de vue sociologique ni même psychologique. Pour moi, ce sont des points de vue qui datent du XIX ${ }^{\mathrm{e}}$ siècle, ils sont complètement dépassés. Je préfere que ma démarche ait un caractère intuitif et poétique.

V. \& I. - Est-ce que vous avez déjà un nouveau projet d'écriture?

J. Poulin - Oui. Je vais essayer d'écrire une autre histoire d'amour.

V. \& I. - Il n'y a pas beaucoup d'histoires d'amour dans vos premiers romans.

J. Poulin - C'est vrai. Quand on commence à écrire, le seul domaine qu'on connaît bien c'est son enfance. C'est là-dessus seulement qu'on peut écrire. Ça prend un certain temps avant qu'on puisse en sortir, et parfois on n'en sort jamais.

V. \& I. - Nous connaissons vos origines beauceronnes. On sait que la Beauce a longtemps conservé une riche tradition orale. Pourrait-on supposer qu'il y a un rapport entre cette tradition orale et le fait que vos livres ont souvent la forme de contes, de récits oraux?

J. Poulin - C'est possible. Chez nous, il y avait une servante qui s'appelait Marie-Ange. Quand nous étions petits, elle nous racontait des histoires que j'ai retrouvées plus tard dans Rabelais. Et puis, au magasin général de mes parents, je me souviens de longues soirées où les gens étaient rassemblés autour d'un grand sac de pinottes en écales et racontaient des histoires de contrebande, parce qu'on était tout près de la frontière américaine. Des histoires, $j$ 'en ai entendu beaucoup dans mon enfance, et j'en ai lu aussi un grand nombre dans l'Encyclopédie de la jeunesse. 
V. \& I. - Dans vos récits, on rencontre beaucoup d'images de dérive par rapport à la navigation, de perte de contrôle par rapport au pilotage automobile. Un passage intrigant dans Volkswagen Blues, c'est quand Jack raconte à la Grande Sauterelle qu'étant enfant, il s'amusait à glisser sur un long ruisseau souterrain gelé. Cette image de glissade dans un tunnel obscur relève peut-être plus de la psychanalyse que de la littérature; néanmoins l'image de vitesse, de dérapage, de perte de contrôle est assez nettement offerte comme analogie à l'écriture.

J. Poulin - Oui, dans plusieurs de mes histoires, le personnage a l'impression qu'il perd le contrôle quand il écrit, qu'il s'en va à la dérive, qu'il ne sait pas trop où ça va le mener. Par rapport au pilotage automobile, c'est la même chose. Et il y a un rapport, comme point commun, avec la sexualité, je suppose. Parce que l'écriture, tout comme le pilotage automobile, c'est un geste très sexuel.

V. \& I. - Un personnage qui suscite beaucoup de réflexion, c'est Théo. II apparaît dans Faites de beaux rêves et revient dans Volkswagen Blues comme une sorte d'alter ego, un homme que le narrateur n'a pas osé être... Est-ce que ce n'est pas un personnage plus ou moins mythique?

J. Poulin - Oui, dans mon esprit, c'était le frère idéal, comme pour Van Gogh, celui qui est un peu plus âgé, celui qui vient à votre aide, le frère universel.

V. \& I. — Et à la fin, il est démythifié...

J. Poulin - La chute des héros est un des thèmes de Volkswagen Blues. Ce roman est plutôt complexe avec plusieurs thèmes qui s'entremêlent: l'écriture comme exploration, la place du français en Amérique, la recherche du paradis perdu, etc. À vrai dire, je n'ai pas très envie de fouiller là-dedans. Et ce n'est pas tellement avec des idées, mais plutôt avec des émotions qu'on écrit des romans.

V. \& I. - Dans une entrevue, il y a quelques années, vous disiez que jusqu'à présent, vous aviez évité les romans à idées, mais que vous aimeriez désormais vous diriger dans cette voie.

J. Poulin - J'ai dû dire ça dans un moment d'aberration...

V. \& I. - Vous avez dit aussi que les Américains faisaient des romans d'action, et les Français des romans d'idées, et que les écrivains du Québec...

J. Poulin - ... et que les écrivains du Québec pouvaient écrire des romans qui feraient la synthèse entre le goût américain pour l'action et le goût français pour les idées. Oui, je me souviens d'avoir dit ça quelque part. Ça semble prétentieux, mais je trouve encore que c'est un projet intéressant. C'est une sorte de rêve québécois!

V. \& I. - Dans Volkswagen Blues, vous nommez des écrivains québécois que vous aimez beaucoup: Gabrielle Roy, Nelligan, Saint-Denys Garneau et Ducharme. Chez ce dernier, il y a un humour, une ironie féroce, qui est tout le contraire de vous. Votre ironie est douce, elle n'est même pas moqueuse.

J. Poulin - J'aime bien Ducharme, mais j'aime bien aussi Boris Vian où il y a le même genre d'ironie assez dure. Ce que j'aime en eux, ce n'est pas leur ironie, mais leur poésie, l'aspect plus doux qu'ils ont aussi. 
V. \& I. - On constate qu'il y a beaucoup plus d'introspection dans les premiers romans et moins par la suite. Vous faites dire à Noël: J'écris pour ne pas me sentir coupable, pour être aimé.

J. Poulin - Je peux résumer cela en disant que dans mes premiers livres, les personnages cherchaient beaucoup d'affection. L'écriture, c'était une façon d'en trouver. Mais au fil des livres, c'est plutôt la recherche de la perfection qui prend de l'importance, le besoin de faire une chose en soi aussi belle que possible.

V. \& I. - Après le troisième roman, la voix narrative passe de la première personne à la troisième personne. Était-ce une tentative de vous distancier du personnage?

J. Poulin - La question du «Je» et du «Il» est pour moi un problème très important. À chaque fois, c'est un cauchemar. Je fais des essais pendant des mois. Je change d'idée, je demande aux autres ce qui convient le mieux. Je n'arrive pas à choisir entre les deux, et pourtant, le résultat est très différent, car lorsque j'utilise le «Il», je m'abstiens d'aller dans la tête du narrateur. Parfois, j'ai adopté une technique spéciale qui consistait à écrire avec un «Je» et de corriger en changeant le «Je» pour un «Il». Plusieurs livres, y compris les Grandes Marées, ont été faits comme ça. Contrairement à la plupart des auteurs, je ne me donne pas la permission de dire: Il pensait que... Avec un «Il», je ne décris que ce que l'on voit de l'extérieur.

V. \& I. - De là, sans doute, l'impression qu'on a d'être en face d'un personnage plutôt froid et indifférent?

J. Poulin - C'est ça. Mon «Il» crée une impression de distance et de froideur. Mais dans le Vieux Chagrin, qui est une histoire plus intime, j'ai hésité moins longtemps que d'habitude et j'ai utilisé le «Je» pour que l'écriture laisse passer autant d'émotions que possible. J'espère que je ne me suis pas trompé.

V. \& I. - Il y a dans vos descriptions une volonté de précision, un besoin de voir exprimer le dehors seulement des choses, mais pas un dehors qui se réfère à un dedans. Plutôt un dehors pur, en fait.

J. Poulin - Oui, absolument, comme si ce n'étaient que les choses pouvant être vues par un observateur neutre. Pour moi, c'est absolument capital. Cela oriente tout mon travail.

V. \& I. - En terminant, Jacques Poulin, votre opinion sur la littérature québécoise actuelle?

J. Poulin - La production est de plus en plus nombreuse et variée. C'est de bon augure. La qualité viendra avec la quantité, car il y a plusieurs jeunes qui ont beaucoup de talent.

Paris, le 12 mai 1989 